\title{
The MiR-495/Annexin A3/P53 Axis Inhibits the Invasion and EMT of Colorectal Cancer Cells
}

\author{
Zhigang Bai Jin Wang Tingting Wang Yuan Li Xiaomu Zhao Guocong Wu \\ Yingchi Yang Wei Deng Zhongtao Zhang
}

Department of General Surgery, Beijing Friendship Hospital, Capital Medical University. Beijing Key Laboratory of Cancer Invasion and Metastasis Research, National Clinical Research Center for Digestive Diseases, Beijing, P. R. China

\section{Key Words}

Colorectal cancer • MiR-495 • Annexin A3 • P53 • Invasion • EMT

\begin{abstract}
Background/Aims: More and more reports have shown that the dysregulation of miRNAs can contribute to the progression and metastasis of human cancers. Many studies have shown that the down-regulation of the miR-495 level occurs in a variety of cancers, including colorectal cancer (CRC). However, the precise molecular mechanisms of miR-495 in CRC have not been well clarified. In the current study, we investigated the biological functions and molecular mechanisms of miR-495 in CRC cell lines. Methods: qRT-PCR was used to determine the level of miR-495 in CRC cell lines and tissues. A miR-495 mimic and inhibitor were transfected into CRC cells, and the effects of miR-495 on the invasion and EMT were explored by qRT-PCR as well as transwell and Western blot assays. Meanwhile, luciferase assays were performed to validate Annexin A3 as a miR-495 target in CRC cells. Results: In our study, we found that miR-495 is down-regulated in CRC tissues and cell lines. Moreover, the low level of miR-495 was associated with increased expression of Annexin A3 in CRC tissues and cell lines. The invasion and EMT of CRC cells were suppressed by the overexpression of miR-495. However, the down-regulation of miR-495 promoted the invasion and EMT of CRC cells. Bioinformatics analysis predicted that Annexin A3 was a potential target gene of miR-495. Next, the luciferase reporter assay confirmed that miR-495 could directly target Annexin A3. Consistent with the effect of miR-495, the down-regulation of Annexin A3 by siRNA inhibited the invasion and EMT of CRC cells through the up-regulation of p53. The introduction of Annexin A3 in CRC cells partially blocked the effects of the miR-495 mimic. Conclusion: The introduction of miR495 directly targeted Annexin A3 to inhibit the invasion and EMT of CRC cells by up-regulating p53, and the down-regulation of Annexin A3 was essential for inhibiting the invasion and EMT of CRC cells by overexpressing miR-495. Overall, the re-activation of the miR-495/Annexin A3/ p53 axis may represent a new strategy for overcoming metastasis of CRC.

Z. Bai and J. Wang contributed equally to this work.

Zhongtao Zhang

Department of General Surgery, Beijing Friendship Hospital, Capital Medical University Beijing Key Laboratory of Cancer Invasion and Metastasis Research, National Clinical Research Center for Digestive Diseases, Beijing (China)

Tel. +86-010-63138745, Fax +86-010-63138745, E-Mail zhangzht@medmail.com.cn
\end{abstract}

KARGER 


\section{Introduction}

Colorectal cancer (CRC), also known as colon cancer, is the third most common cancer and the second leading cause of cancer-related death worldwide [1]. CRC is a common malignant neoplasm in the digestive tract. Moreover, the invasion and metastasis of cancer are the main causes of mortality in patients with CRC [2]. During the development of CRC, the activation of oncogenes and inactivation of suppressors play critical roles in invasion and metastasis [3]. Although treatment has improved, $50 \%$ of patients with CRC still develop metastases after surgery [4]. The precise molecular mechanisms underlying the invasion and metastasis of CRC remain largely unknown. Therefore, it is highly important to identify new predictive biomarkers and to understand the precise mechanisms for the invasion and metastasis of CRC.

Annexins are a structurally homologous family of calcium-dependent phospholipidbinding proteins [5]. The 12 human Annexins play different roles in regulating the activity of phospholipase A2, membrane trafficking, cell division, differentiation, apoptosis and blood coagulation [5-8]. In addition, Annexins play critical roles in carcinogenesis [9]. Recent reports show that the up-regulation and down-regulation of Annexin members in multiple cancers are closely associated with the progression of cancers [9]. However, the expressions of Annexins, such as A1, A2, A3, A5 and A7, in CRC are still unclear.

Many studies have reported that microRNAs (miRNAs) play important roles in regulating biological and pathologic processes, including invasion and metastasis [10]. They generally function as crucial gene regulators. Recently, several reports have showed that miRNAs are involved in metastasis and tumorigenesis by targeting many types of mRNAs [11]. To date, the dysregulated expression of multiple miRNAs, such as miR-4775 [12], miR-30a-5p [13], and miR-124 [14], have been demonstrated to contribute to the invasion and metastasis of CRC. These findings provide a strong basis for the importance of miRNAs in the pathogenesis of CRC and emphasize the implications of miRNAs in the diagnosis, therapy, and prognosis of CRC.

In this study, the level of Annexin A3 was found to be the highest among Annexins in CRC tissues in comparison to the adjacent tissues. Furthermore, we found that miR-495 could directly target Annexin A3 in CRC cells using the online database TargetScan 6.2. Currently, miR-495 has attracted much attention because several studies have reported that miR-495 is frequently decreased and functions as a tumor suppressor in gliomas as well as gastric and endometrial cancers [15-17], but it is increased and functions as an oncogene in breast and bladder cancers $[18,19]$. However, until now, the level of miR-495 and its the precise mechanism in CRC have been unclear. We first found that the level of miR-495 was frequently down-regulated in CRC tissues and cell lines. The introduction of miR-495 suppressed the invasion and epithelial-mesenchymal transition (EMT) of CRC cells. The overexpression of Annexin A3 reversed the inhibitory effects of a miR-495 mimic on CRC cells through the down-regulation of p53. Hence, our results showed important roles for miR-495 in the pathogenesis of CRC and suggested its potential application in its treatment.

\section{Materials and Methods}

\section{Cell culture and human tissues}

The human CRC cell lines SW620, SW480, HT29, HCT116 and human normal colon epithelium cell line FHC were purchased from the American Type Culture Collection (ATCC, Manassas, VA, USA). The cells were cultured in Dulbecco's modified Eagle's medium (DMEM) (Gibco Co., New York, USA) containing 10\% fetal bovine serum (FBS) (Gibco Co., New York, USA), $100 \mathrm{mg} / \mathrm{ml}$ penicillin and $100 \mathrm{mg} / \mathrm{ml}$ streptomycin at 37 ${ }^{\circ} \mathrm{C}$ in a humidified atmosphere of $5 \%$ on $0.1 \%$ gelatin-coated culture flasks. Twenty pairs of human primary CRC tissues and their adjacent normal tissues were collected at Beijing Friendship Hospital of Capital Medical University. Patients recruited in this study were newly diagnosed with CRC and had not received any treatment. The tumor and adjacent normal tissues were obtained after surgical resection, immediately frozen in liquid nitrogen and then stored at $-80^{\circ} \mathrm{C}$ for analysis. Prior informed consent was obtained, and the 


\begin{tabular}{|c|c|}
\hline Cellular Physiology & Cell Physiol Biochem 2017;44:1882-1895 \\
\hline emistry & 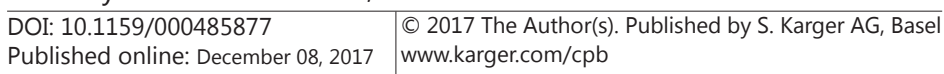 \\
\hline
\end{tabular}

study protocol was approved by the Ethics Committee of Beijing Friendship Hospital of Capital Medical University. The pathological characteristics of patients are shown in Table 1.

\section{MiRNA transfection}

To up-regulate or downregulate the level of miR-495, HCT116 cells were transfected with a miR-495 mimic and a miR-negative control (miRNC) or a miR-495 inhibitor and a miR-negative control for the inhibitor (inhibitorNC) that were synthesized by Gene-Pharma (Shanghai, China). The transfections were done using Lipofectamin 3000 reagent (Invitrogen, USA) according to manufacturer's instructions. The pcDNA3.1 vector, pcDNA-Annexin A3, siRNA for Annexin A3 (si-Annexin A3), si-p53 and siRNA-negative control (si-NC) were synthesized and purified by Gene-Pharma (Shanghai, China).

Reverse transcription polymerase chain reaction

The total RNA of the HCT116 cells was extracted by using TRIzol reagent (Qiagen, USA) in order to analyze the miRNA and mRNA levels according to the manufacturer's protocols. For quantification of miR-495, the TaqMan MicroRNA Reverse Transcription Kit and the TaqMan miRNA assay (Applied Biosystems, USA) were used to perform reverse transcription and PCR according to the manufacturer's instructions. U6 was used as an internal control. The gene expressions of Annexin A3, E-cadherin, $\beta$-catenin, fibronection, vimentin, Snail, Slug, ZEB1 and ZEB2 were detected using SYBR Green PCR kits (Qiagen, USA). GAPDH served as an internal control. U6 snRNA and GAPDH mRNA were used to normalize the data. The primers used are listed in Table 2.

Transwell invasion assay

A transwell Matrigel invasion assay using transwell chambers (8- $\mu \mathrm{m}$ pore size; Minipore) precoated with Matrigel
Table 2. Sequence of the primers used for qRT-PCR

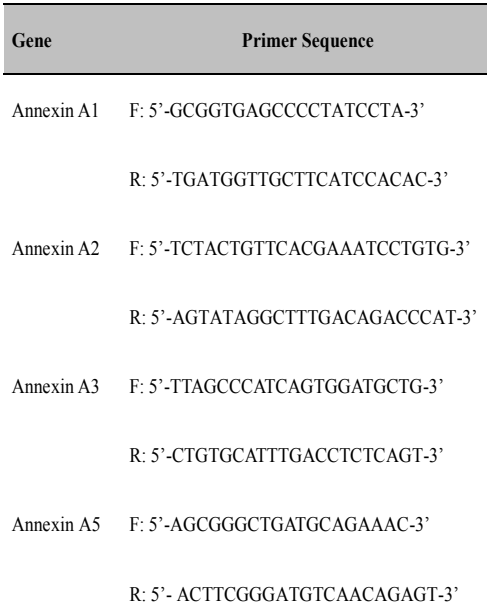

Annexin A7 F: 5'-CCTAGTGGCTTTCCTCCAATG-3'

R: 5'- ACCAGGATAGCCTCCAGGG-3'

E-cadherin F: 5'-TACACTGCCCAGGAGCCAGA-3'

R: 5'-TGGCACCAGTGTCCGGATTA-3'

ß-catenin F: 5'-AAAGCGGCTGTTAGTCACTGG-3'

R: 5'-CGAGTCATTGCATACTGTCCAT-3'

fibronection F: 5'-CGGTGGCTGTCAGTCAAAG-3'

R: 5'-AAACCTCGGCTTCCTCCATAA-3'

vimentin F: 5'-GACGCCATCAACACCGAGTT-3'

R: 5'-CTTTGTCGTTGGTTAGCTGGT-3'

Snail F: 5'-TCGGAAGCCTAACTACAGCGA-3,

R: 5'-AGATGAGCATTGGCAGCGAG-3'

Slug F: 5'-CGAACTGGACACACATACAGTG-3'

R: 5'-CTGAGGATCTCTGGTTGTGGT-3',

ZEB1

F: 5'-GATGATGAATGCGAGTCAGATGC-3'

F: 5'-ACAGCAGTGTCTTGTTGTTGT-3'

ZEB2 F: 5'-CAAGAGGCGCAAACAAGCC-3'

F: 5'-GGTTGGCAATACCGTCATCC-3'

U6 F: 5'-CTCGCTTCGGCAGCACA-3'

F. 5'-AACGCTTCACGAATTTGCGT-3'

GAPDH F: 5'-GAGTCAACGGATTTGGTCGTATTG-3'

R: 5'-CCTGGAAGATGGTGATGGGATT-3' 


\section{Cellular Physiology Cell Physiol Biochem 2017;44:1882-1895 \begin{tabular}{l|l|l} 
and Biochemistry 10.1159/000485877 & $\begin{array}{l}\text { (c) 2017 The Author(s). Published by S. Karger AG, Basel } \\
\text { www.karger.com/cpb }\end{array}$
\end{tabular}}

Bai et al.: The Roles of MiR-495 in Colorectal Cancer

(BD Biosciences, Franklin Lakes, NJ) that contained extracellular matrix proteins was used to assess cell invasion. In brief, $1 \times 10^{5}$ cells in $100 \mu \mathrm{l}$ DMEM containing $1 \%$ FBS were seeded in the upper chamber, and $600 \mu \mathrm{l}$ DMEM containing 10\% FBS were added to the lower chamber. After $24 \mathrm{~h}$ of incubation at $37{ }^{\circ} \mathrm{C}$ in a $5 \% \mathrm{CO}_{2}$ atmosphere, cells that remained in the upper chamber were removed by cotton swabs, and the penetrating cells were fixed in methanol and then stained with $0.1 \%$ crystal violet. Then, the membranes were rinsed with $30 \%$ glacial acetic acid. Finally, the absorbance of the washing solution was assessed at $540 \mathrm{~nm}$ for cell counting measurements.

Measurement of the matrix metalloproteinase (MMP)-2, MMP-7, MMP-9, tissue inhibitor of metalloproteinase (TIMP)-1 and TIMP-2 levels

Enzyme-linked immunosorbent assay (ELISA) kits (USCN, USCN Life Science, Wuhan, China) were used to determine the levels of MMP-2, MMP-7, MMP-9, TIMP-1 and TIMP-2 in the culture supernatants based on the manufacturer's instructions.

\section{Western blot analysis}

HCT116 cells were washed twice in cold PBS and then lysed in RIPA lysis buffer (Beyotime Institute of Biotechnology Jiangsu, China) containing a protease inhibitor cocktail (Merk, USA). The protein concentration of the cell lysates was quantified using a BCA Kit (Beyotime Institute of Biotechnology Jiangsu, China), and 50 ng of protein for each sample were separated by SDS-PAGE on 10\% gels and then transferred to a polyvinylidene fluoride (PVDF) membrane (Millipore, USA). The membranes were blocked in 5\% non-fat milk diluted with TBST at room temperature for $1 \mathrm{~h}$ and incubated overnight at $4{ }^{\circ} \mathrm{C}$ with primary antibodies, including anti-Annexin A3, anti-MMP-7, anti-MMP-9, anti-E-cadherin, anti- $\beta$-catenin, anti-fibronection, anti-vimentin, anti-Snail, anti-Slug, anti-ZEB1 and anti-ZEB2 (1:500; Abcam, USA) as well as anti-MMP-2, anti-TIMP-1, anti-TIMP-2 and anti-p53 (1:1000; Cell Signaling Technology Inc., USA). The membranes were then incubated with a goat anti-rabbit or anti-mouse IgG conjugated to horseradish peroxidase secondary antibody (1:1000; Cell Signaling Technology Inc., MA, USA) for $2 \mathrm{~h}$. The proteins were visualized using ECL Plus reagents (Amersham Biosciences Corp., USA). The density of the bands was measured using the Image J software (USA), and the values were normalized to the densitometric values of $\alpha$-tubulin (1:1000; Sigma, USA) or $\beta$-actin (1:5000; Sigma, USA) in each sample.

\section{Luciferase reporter assay}

HCT116 cells were seeded in 24-well plates and incubated for $24 \mathrm{~h}$ before transfection. The pMIRAnnexin A3-3'UTR wild-type or mutant plasmid was cotransfected with a miR-495 mimic or miR-NC and the pRL-TK plasmid (Promega, USA) into HCT116 cells. After transfection for $24 \mathrm{~h}$, a luciferase reporter gene assay was implemented using the dual-luciferase reporter system (Promega, USA) following the manufacturer's protocols. All experiments were performed at least 3 times.

\section{Statistical analysis}

All statistical analyses were performed using the GraphPad Prism 5.0 software (GraphPad Software, Inc., USA). Data from each group were expressed as the mean \pm standard error of the mean (S.E.M.). Student's t-test was used to analyze differences between two groups. A one-way ANOVA was performed to detect statistical differences among multiple groups. Differences within the ANOVA were determined using a Tukey's post hoc test. Differences were considered statistically significant at a $\mathrm{p}$ value of $<0.05$.

\section{Results}

The expression of Annexin A3 was up-regulated and the level of miR-495 was downregulated in $C R C$ tissues and cell lines

It has been reported that Annexins, such as Annexin A1, A2 A3, A5 and A7, are closely associated with cancers [20]. In this study, we detected these five Annexins genes in CRC tissues. The results showed that the mRNA level of Annexin A3 was the highest in CRC tissues among these five Annexins genes compared with the adjacent tissues (Fig. 1A). Furthermore, we detected the expression of Annexin A3 for stage I/II and stage III. Our results showed 


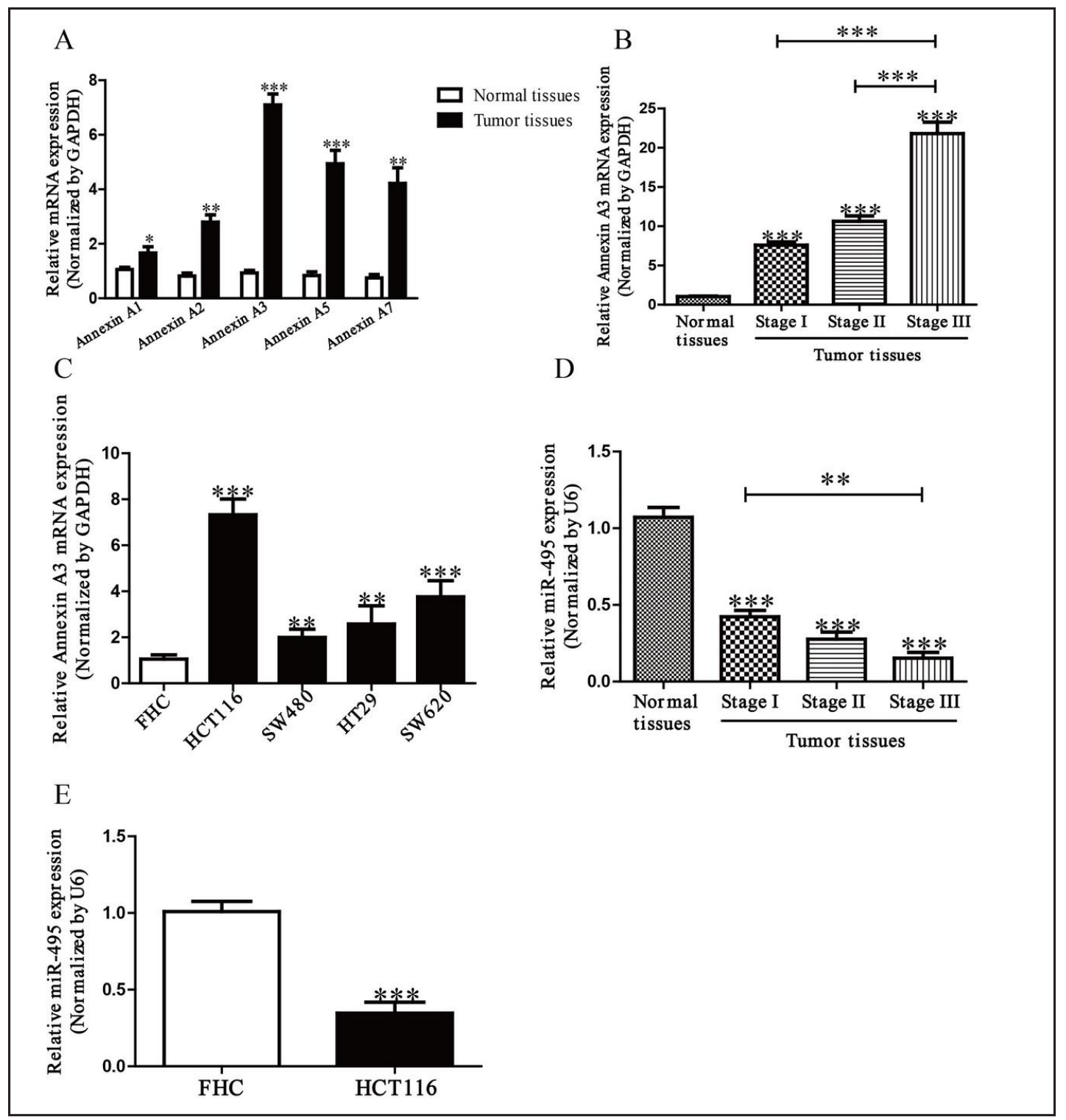

Fig. 1. The levels of Annexin A3 and miR-495 in CRC tissues and cell lines. (A) The mRNA levels of Annexin A1, A2, A3, A5 and A7 in CRC tissues and their corresponding adjacent normal tissues. (B) The expression of Annexin A3 in stage I, II and III CRC tissues. (C) The mRNA expression of Annexin A3 analyzed by qRT-PCR in four CRC cell lines (HCT116, SW480, HT29 and SW620) and a normal colon epithelium cell line (FHC). (D) The relative miR-495 level in stage I, II, and III CRC tissues and their corresponding adjacent normal tissues. (E) The relative miR-495 level analyzed by qRT-PCR in HCT116 cells and a normal colon epithelium cell line (FHC). All data are presented as the mean \pm SEM, $\mathrm{n}=6 .{ }^{*} \mathrm{P}<0.05,{ }^{* *} \mathrm{P}<0.01,{ }^{* * *} \mathrm{P}<0.001$ vs. Normal, stage I, stage II CRC tissues or FHC.

that the expression of Annexin A3 in stage III CRC tissues was expressed higher than that in stage I or II CRC tissues (Fig. 1B). Subsequently, we also determined the mRNA level of Annexin A3 in four CRC cell lines (HCT116, SW480, HT29 and SW620) and a normal colon epithelium cell line (FHC). Compared with the FHC line, the level of Annexin A3 in HCT116 cells was higher than that in the other three CRC cell lines (Fig. 1C). Thus, HCT116 cells were used in the following experiments. For further study, we found that miR-495 may directly target Annexin A3 using the online database TargetScan 6.2. Next, our results showed that the level of miR-495 in the CRC tissues was significantly lower in comparison to the adjacent 
Fig. 2. The effects of miR-495 on the invasion and the expression of related molecules in HCT116 cells. HCT116 cells were transfected with a miR-495 mimic or an inhibitor. (A) The level of miR-495 analyzed by qRT-PCR. (B) The invasion of HCT116 cells was assessed by a transwell assay. (C) and (D) The levels of total MMP-2, MMP-7, MMP-9 and TIMP-2 were detected in the culture supernatants of cultured HCT116 cells with an ELISA. (E) and (F) The protein expressions of MMP2, MMP-7, MMP-9, TIMP-1 and TIMP-2 were examined by Western blot. All data are presented as the mean \pm SEM, $n=4 .{ }^{*} \mathrm{P}<0.05$, ${ }^{\# \#} \mathrm{P}<0.01$, ${ }^{\# \# \# P} \mathrm{P}<0.001$ vs. miR-NC or inhibitor-NC.
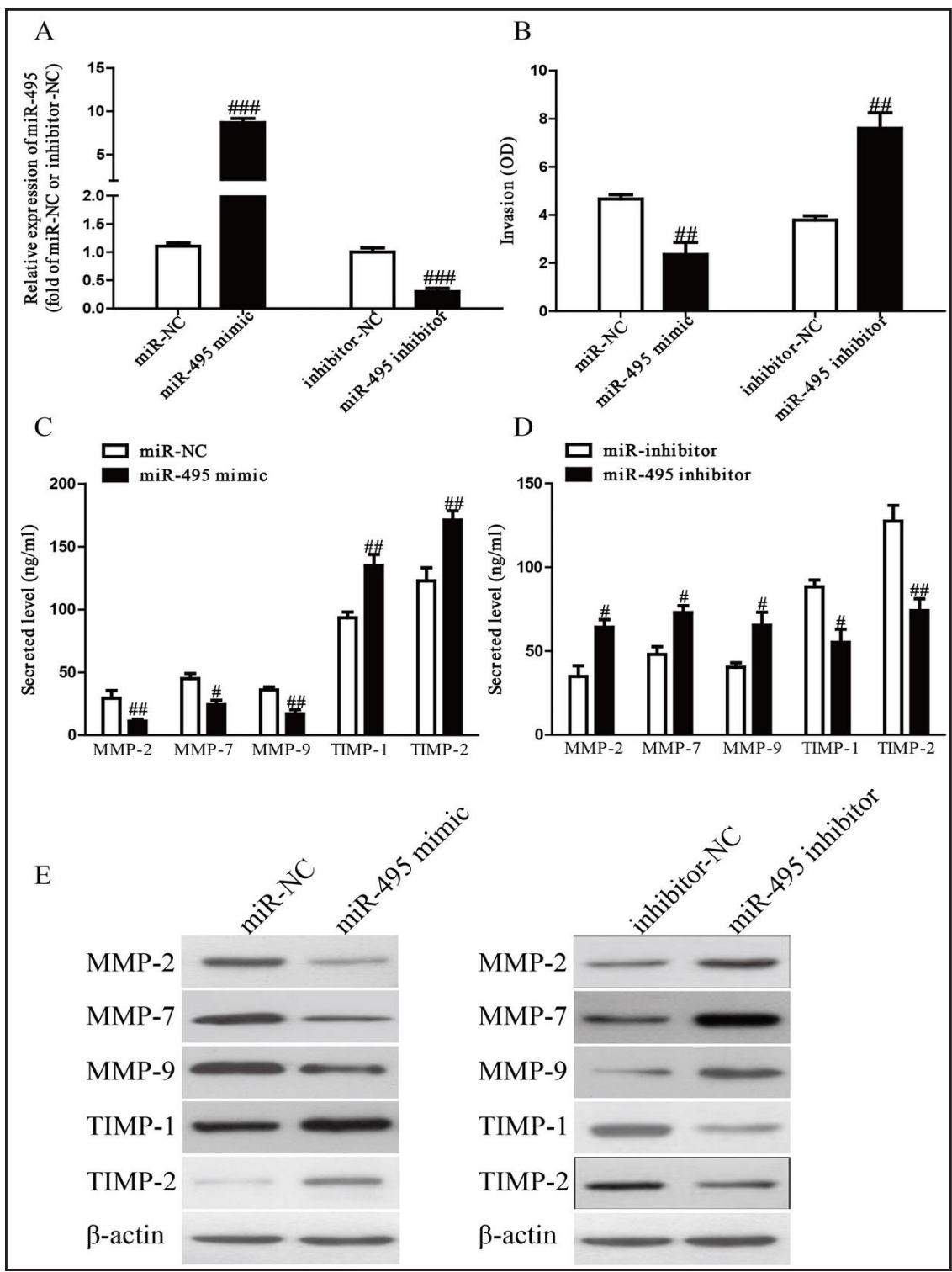

tissues, and the level of miR-495 in stage III CRC tissues expressed lower than that in stage I CRC tissues (Fig. 1D). To confirm this result, we also demonstrated that miR-495 expression was markedly down-regulated in HCT116 cells compared to that in FHC, as shown in Fig. 1E.

\section{Effect of miR-495 on the invasion and related molecules of CRC cells}

After transfection with a miR-495 mimic and an inhibitor, the qRT-PCR analysis showed that the level of miR-495 was significantly up-regulated and down-regulated, respectively (Fig. 2A). These data demonstrated that we efficiently enhanced and reduced miR-495 expression, respectively, in HCT116 cells. To study the role of miR-495 in the invasion of CRC cells, we evaluated the invasive capacities of HCT116 cells transfected with a miR495 mimic or an inhibitor using transwell invasion assays. The transwell assays illustrated that the invasion of HCT116 cells was remarkably suppressed in the miR-495 mimic group compared to the miR-NC group but was significantly promoted in the miR-495 inhibitor group compared to the anti-miR-NC group (Fig. 2B). These findings showed that miR-495 might play a critical role in the inhibition of CRC cell invasion. The balance between MMPs and TIMP-2 is demonstrated to play an important role of invasion by stimulating the degradation 
Fig. 3. The effects of miR-495 on expressions of EMT-related molecules in HCT116 cells. (A) The mRNA and protein levels of E-cadherin, $\beta$-catenin, fibronection and vimentin were determined by qRT-PCR and Western blot in HCT116 cells transfected with a miR-495 mimic or an inhibitor. (B) The mRNA and protein levels of Snail, Slug, ZEB1 and ZEB2 were determined by qRT-PCR and Western blot in HCT116 cells transfected with a miR-495 mimic or an inhibitor. All data are presented as the mean \pm SEM, $n=4$. ${ }^{\#} \mathrm{P}<0.05$, \#\#P<0.01 vs. miR-NC or inhibitor-NC.
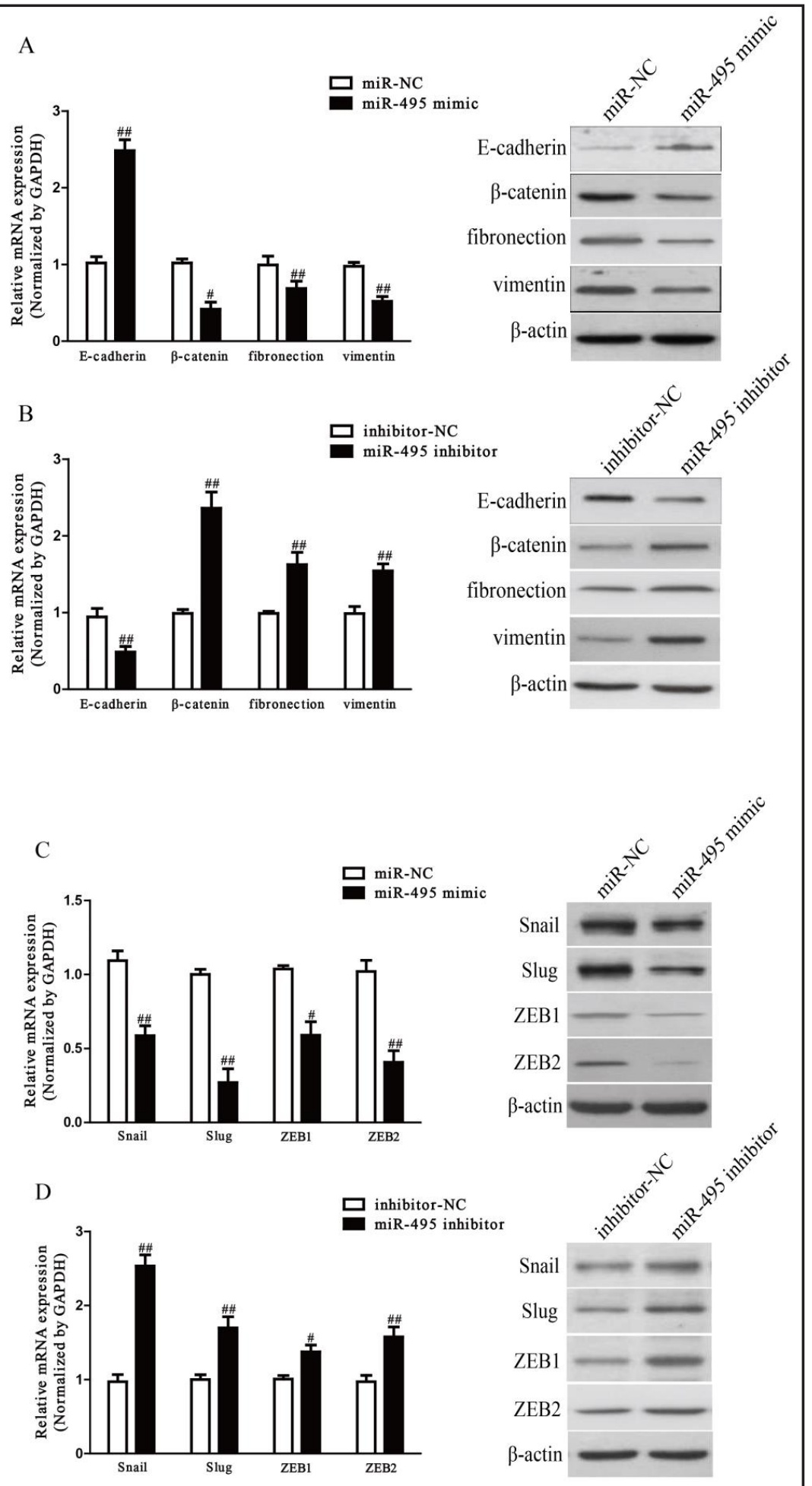

of the ECM in CRC cells and is associated with enhanced tumor metastatic potential. Our ELISA and Western blot assays indicated that the total secretion of MMP-2, MMP-7, and MMP-9 in the culture supernatants and the expressions of MMP-2, MMP-7, and MMP-9 were evidently decreased by the overexpression of miR-495 in HCT116 cells and the total secretion of TMIP-1, TIMP-2 and protein expressions of TMIP-1, TIMP-2 were significantly increased (Fig. 2C, D, E). However, the knockdown of miR-495 could enhance the secretion 
$\mathrm{A}$

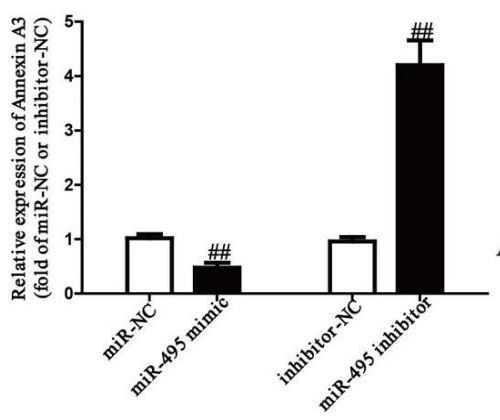

$\mathrm{C}$

hsa-miR-495

Annexin A3 3'UTR WT

Annexin A3 3'UTR MUT
B

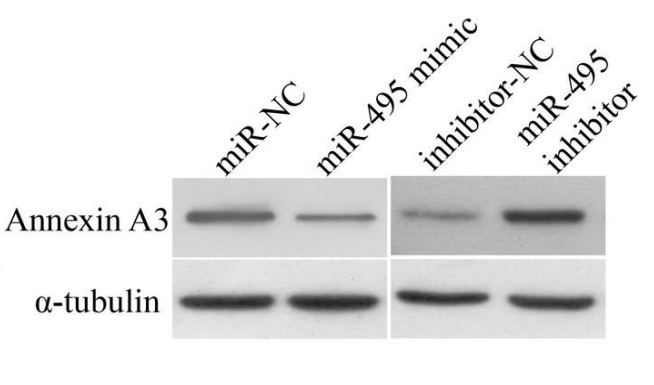

3' uucuucacgugguacAAACAAa

5, auaaaaauuguugcuUUUGUUaa

5' auaaaaauuguugcuAAACAAaa
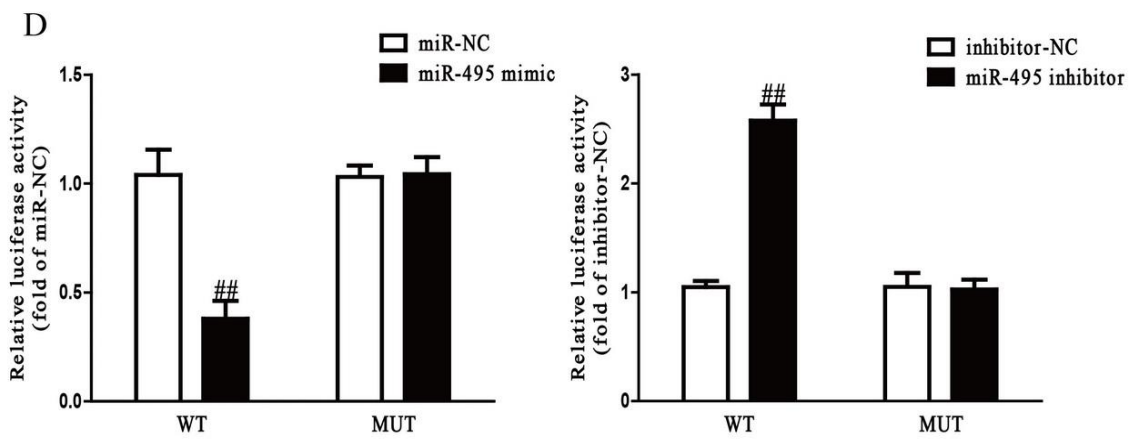

Fig. 4. Annexin A3 is a direct target of miR-495. (A) The mRNA level of Annexin A3 was determined by qRTPCR in HCT116 cells transfected with a miR-495 mimic or an inhibitor. (B) The protein expression of Annexin A3 was determined by Western blot in HCT116 transfected with a miR-495 mimic or an inhibitor. (C) A schematic representation of Annexin A3 3'UTRs showing the putative miRNA target site. (D) The analysis of the relative luciferase activities of Annexin A3-WT, Annexin A3-MUT in CRC cells. All data are presented as the mean $\pm \mathrm{SEM}, \mathrm{n}=4 .{ }^{\# \#} \mathrm{P}<0.01$ vs. miR-NC or inhibitor-NC.

and protein expressions of MMP-2, MMP-7, and MMP-9 and reduce the secretion and protein expressions of TMIP-1 and TMIP-2 (Fig. 2D, F). Taken together, our findings suggested that the down-regulation of MMP-2, MMP-7, and MMP-9 and the up-regulation of TMIP-1 and TMIP-2 might be the possible mechanisms contributing to the inhibitory effect of the miR495 mimic on the invasion of HCT116 cells.

\section{Effects of miR-495 on EMT of CRC cells}

Next, we explored the effects of miR-495 on the expressions of EMT markers in HCT116 cells using qRT-PCR and Western blot. The introduction of miR-495 in HCT116 cells increased the expression of the epithelial marker E-cadherin and decreased the expressions of mesenchymal markers $\beta$-catenin, fibronection and vimentin at both the mRNA and protein levels (Fig. 3A). Moreover, we also determined the expressions of EMT-related transcription factors in HCT116 cells after transfection with a miR-495 mimic. The overexpression of miR495 significantly reduced the mRNA and protein expressions of Snail, Slug, ZEB1 and ZEB2 
Fig. 5. Annexin $\mathrm{A} 3$ silencing could also inhibit the invasion and EMT of CRC cells. HCT116 cells were transfected with si-Annexin A3 or si-p53. (A) The mRNA and protein levels of Annexin A3 were determined by qRT-PCR and Western blot, respectively. (B) The mRNA and protein levels of p53 were determined by qRTPCR and Western blot, respectively. (C) The invasion of HCT116 cells was assessed by a transwell assay. (D) The levels of total MMP-2, MMP7, MMP-9, TIMP-1 and TIMP-2 were
A
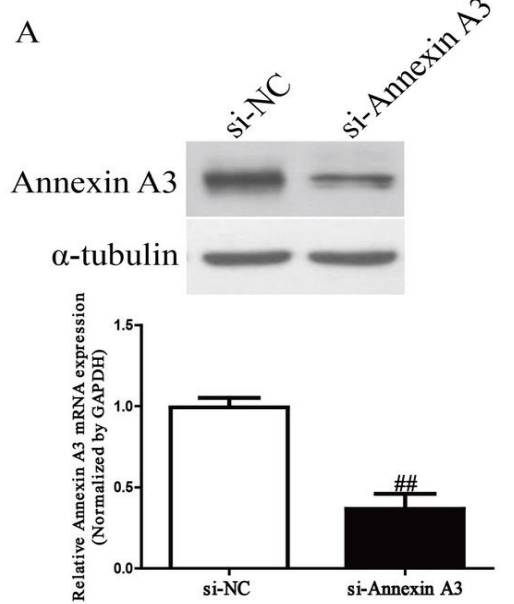

$\mathrm{C}$

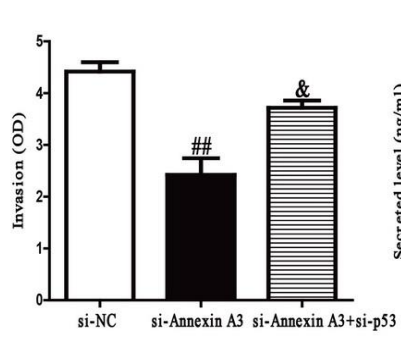

D
B
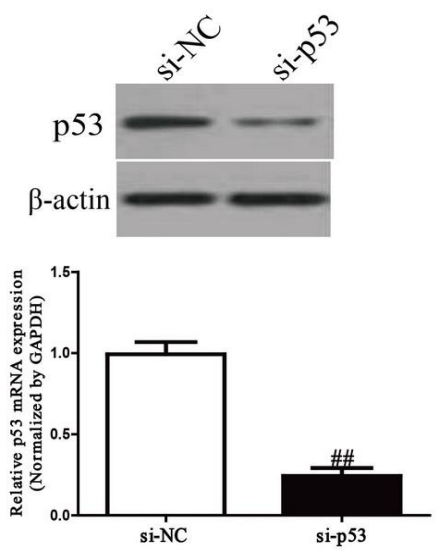
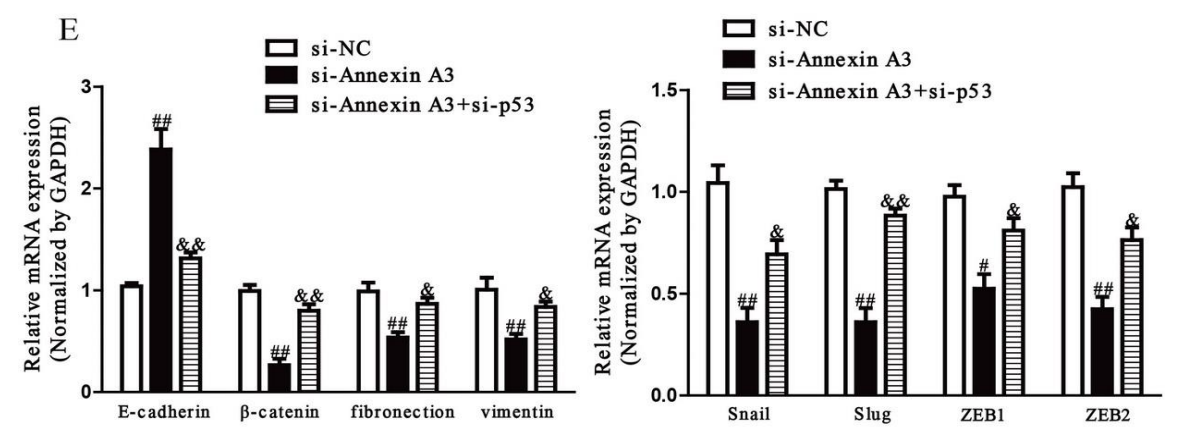

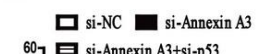

${ }^{60} 7$ 吕 si-Annexin A3+si-p53
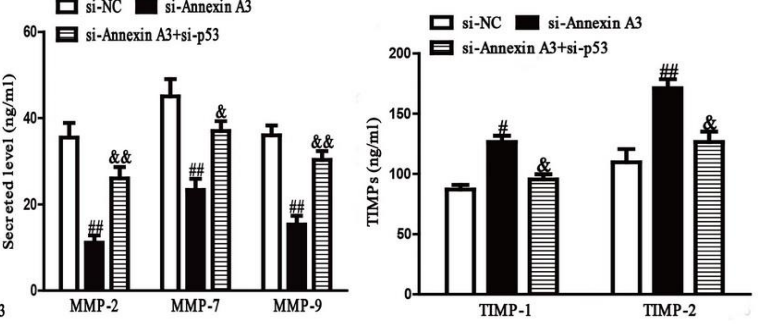

detected in the supernatants of cultured HCT116 cells using an ELISA. (E) The expressions of E-cadherin, $\beta$-catenin, fibronection, vimentin, Snail, Slug, ZEB1 and ZEB2 were determined by qRT-PCR in HCT116 cells. All data are presented as the mean \pm SEM, $n=4$. ${ }^{\#} \mathrm{P}<0.05$, ${ }^{\# \#} \mathrm{P}<0.01$, vs. si-NC; ${ }^{\mathrm{P}} \mathrm{P}<0.05$, ${ }^{\&} \mathrm{P}<0.01$ vs. si-Annexin A3.

in both cells (Fig. 3C). However, the knockdown of miR-495 had opposite effects on miR-495 up-regulation in the EMT of CRC cells (Fig. 3B, D). Altogether, our findings revealed that the overexpression of miR-495 could inhibit the EMT of CRC cells.

miR-495 directly targets Annexin A3 in CRC cells

Based on the TargetScan 6.2 database, we predicted that Annexin A3 was a binding target of miR-495. Next, we performed qRT-PCR and Western blotting to assess the expression of Annexin A3 at the mRNA and protein levels in HCT116 cells transfected with a miR-495 mimic or inhibitor. Our findings showed that the mRNA and protein levels of Annexin A3 were significantly decreased after transfection with a miR-495 mimic and were dramatically increased after transfection with a miR-495 inhibitor (Fig. 4A and B). Moreover, a luciferase reporter assay also confirmed that miR-495 directly targeted Annexin A3. Annexin A3 3'- 
Fig. 6. The overexpression of Annexin A3 partially rescued miR495-inhibited invasion and EMT in CRC cells. HCT116 cells were transfected with either a miR-495 mimic with or without the pcDNA-Annexin A3 vector. (A) The mRNA and protein levels of Annexin A3 were determined by qRT-PCR and Western blot, respectively.

(B) The invasion of HCT116 cells was assessed by a transwell assay. (C) The levels of total MMP-2, MMP-7, MMP-9, TIMP-1 and TIMP2 were detected in the supernatants of cultured HCT116 cells by an ELISA. (D) The expressions of E-cadherin, $\beta$-catenin, fibronection and vi-
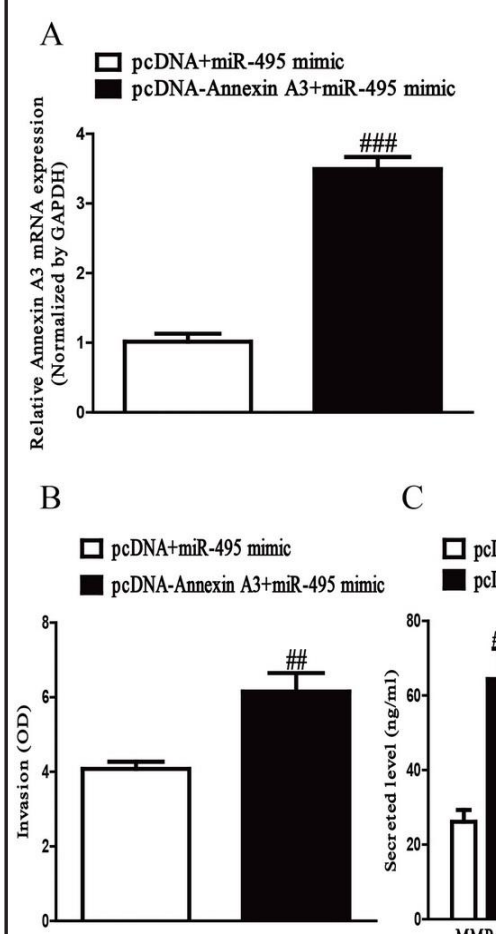

$\square$ pcDNA+miR-495 mimic $\square$ pcDNA-Annexin A3+miR-495 mimic miR-495 mimic pcDNA pcDNA-Annexin A3

Annexin A3 $\alpha$-tubulin
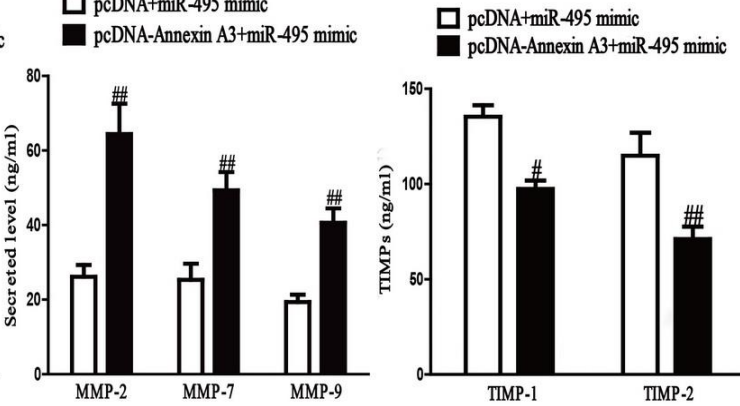

$\mathrm{D}$

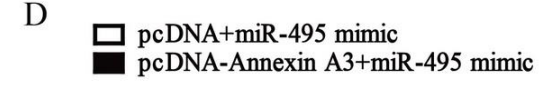

E

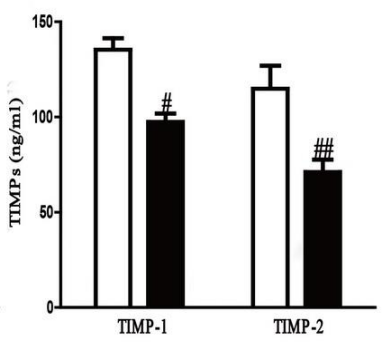

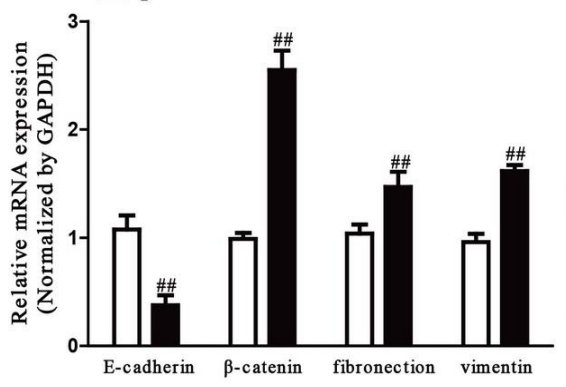

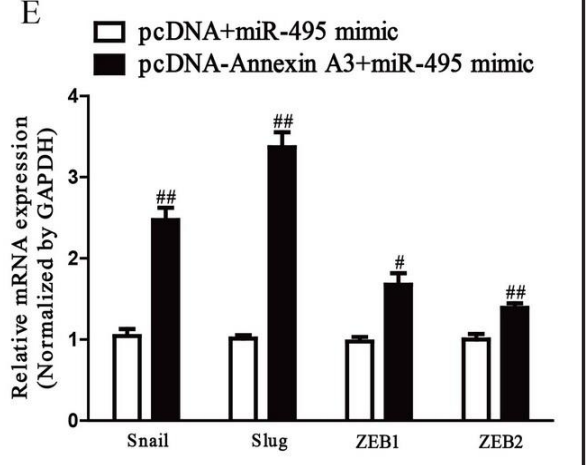

mentin were de-

termined by qRT-PCR in HCT116 cells. (E) The expressions of Snail, Slug, ZEB1 and ZEB2 were determined

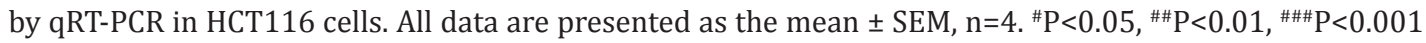
vs. miR-495 mimic + pcDNA.

UTR was cloned into a luciferase reporter vector, and the putative miR-495 binding site in the Annexin A3 3'-UTR was mutated (Fig. 4C). Our data showed that the up-regulation of miR-495 markedly inhibited the luciferase activity of pMIR-Annexin A3 3'-UTR WT (Fig. 4D). The mutation of the miR-495-binding site in the Annexin A3 3'-UTR abolished the effect of miR-495, which indicated that miR-495 directly and negatively regulated the expression of Annexin A3.

Knockdown of Annexin A3 mimicked the effects of the overexpression of miR-495 through the up-regulation of p53

To explore the role of Annexin A3 in CRC cells, HCT116 cells were transfected with siNC or si-Annexin A3. The qRT-PCR and Western blot analysis showed that the expression 


\section{Cellular Physiology Cell Physiol Biochem 2017;44:1882-1895 \begin{tabular}{ll|l} 
and Biochemistry Published onlIne: December 08, 2017 & $\begin{array}{l}\text { (c) 2017 The Author(s). Published by S. Karger AG, Basel } \\
\text { www.karger.com/cpb }\end{array}$ \\
\hline
\end{tabular} \\ Bai et al.: The Roles of MiR-495 in Colorectal Cancer}

of Annexin A3 was significantly decreased at the mRNA and protein level in HCT116 cells transfected with si-Annexin A3 (Fig. 5A). A transwell assay revealed that the down-regulation of Annexin A3 suppressed the invasion of CRC cells (Fig. 5B). Moreover, decreased Annexin A3 expression could significantly reduce the total expression of MMP-2 and enhance the expressions of TMIP-1 and TIMP-2 in HCT116 cells (Fig. 5C). In addition, the silencing of Annexin A3 contributed to the up-regulation of E-cadherin and the down-regulation of $\beta$-catenin, fibronection and vimentin (Fig. 5D). Next, the mRNA levels of Snail, Slug, ZEB1 and ZEB2 in HCT116 cells were also significantly reduced by decreasing the miR-495 level (Fig. 5E). Hence, the knockdown of Annexin A3 induced a very similar phenotype as miR-495 overexpression in CRC cells. In further study, the down-regulation of p53 could significantly block the effects of Annexin A3 inhibition (Fig. 5B-F). Altogether, our data demonstrated that the down-regulation of Annexin A3 inhibited cell invasion and the EMT of CRC through the down-regulation of p53.

MiR-495 suppressed the invasion and EMT of CRC cells through its inhibition of Annexin A3

To confirm whether miR-495 inhibited the invasion and EMT of CRC cells through an Annexin A3-dependent mechanism, we cotransfected HCT116 cells with a miR-495 mimic and the pcDNA3.1-Annexin A3 vector. Our data showed that the expressions of Annexin A3 at the mRNA and protein levels were dramatically increased after transfection with the miR495 mimic and pcDNA-Annexin A3 compared with the miR-495 mimic and a pcDNA vector in HCT116 cells (Fig. 6A). The data from the transwell assay indicated that the up-regulation of Annexin A3 could reverse the inhibitory effect of miR-495 overexpression on the invasion of CRC cells (Fig. 6C). Moreover, the introduction of Annexin A3 decreased the expression of E-cadherin and increased the expressions of $\beta$-catenin, fibronection and vimentin in HCT116 cells after transfection with the miR-495 mimic (Fig. 6D). Next, the up-regulation of Annexin A3 could also reverse the effects of the miR-495 mimic on the expressions of Snail, Slug, ZEB1 and ZEB2 (Fig. 6E). Therefore, our data clearly confirmed that the up-regulation of miR-495 inhibited the p53-regulated invasion and EMT of CRC cells by directly targeting Annexin A3 and the knockdown of Annexin A3 was essential for the miR-495 mimic-induced inhibition of the invasion and EMT in CRC cells.

\section{Discussion}

CRC is the fourth and third most common cancer in males and females, respectively, with over 1.2 million cases diagnosed each year worldwide and approximately 600, 000 deaths [2]. The primary cause of death induced by CRC is metastasis to the liver [21]. Therefore, understanding the precise molecular mechanisms underlying CRC metastasis is critical to the development of novel and effective therapeutic strategies for patients with advanced CRC. Despite structural similarities among the members of this family, Annexin proteins play different roles in anti-inflammation actions, anti-fibrinolytic activity, vesicle trafficking, cell proliferation, apoptosis, differentiation and angiogenesis $[5,6,8,22]$. Recent studies have demonstrated that several forms of annexins are closely associated with the development and progression of cancer, and the expression patterns of individual annexins are tumortype specific [9]. Several annexin proteins, including Annexin A1, A2, A3, A4, A5, A6, A7 and $A 11$, are involved in the oncogenic process. In this study, for the first time, we demonstrated that Annexin A3 had the highest expression in CRC tissues among these annexin proteins. Then, we found that the expression of Annexin A3 in stage III CRC tissues was higher than that in stage I and II CRC tissues. Next, our findings showed that miR-495 was frequently down-regulated in CRC cell lines and tissues. Moreover, the level of miR-495 in stage III CRC tissues was lower than that in stage II CRC tissues. As expected, the introduction of miR-495 inhibited the invasion and EMT of HCT116 cells. Our findings suggested that miR-495 plays crucial roles in regulating the invasion and EMT of CRC and might be a possible diagnostic and predictive biomarker. 


\section{Cellular Physiology Cell Physiol Biochem 2017;44:1882-1895 \begin{tabular}{ll|l} 
and Biochemistry Published onlıne: December 08, 2017 & $\begin{array}{l}\text { (c) } 2017 \text { The Author(s). Published by S. Karger AG, Basel } \\
\text { www.karger.com/cpb }\end{array}$ \\
\hline
\end{tabular} \\ Bai et al.: The Roles of MiR-495 in Colorectal Cancer}

Previously, the precise mechanism of miR-495 in CRC was unclear. Therefore, in this study, we aimed to elucidate the biological functions and its mechanism of miR-495 in CRC. Our qRT-PCR, Western blotting and luciferase reporter assays confirmed that miR-495 directly targeted Annexin A3. Importantly, we also demonstrated that the overexpression of Annexin A3 partly blocked the inhibitory effects of a miR-495 mimic on the invasion and EMT of CRC cells through down-regulation of p53. Therefore, we made the conclusion that miR-495 plays critical roles in the inhibition of the invasion and EMT of CRC cells through the regulation of Annexin $\mathrm{A} 3$ and $\mathrm{p} 53$.

One of the key molecular steps in the process of invasion is the degradation of extracellular matrix (ECM) components by proteolytic enzymes [23]. A previous study showed that the imbalance between MMP activity and the specific inhibitor TIMPs in CRC tissue might be a significant factor in the process of tumor invasion and metastasis [24]. It has been demonstrated that the expression of MMP-2 in CRC tissues was significantly higher, but TIMP-2 was significantly lower than that in normal tissues [24]. In this paper, the transwell assay showed that the overexpression or knockdown of miR-495 dramatically suppressed or promoted the invasion of HCT116 cells compared with the miR-NC or anti-miR-NC group, respectively. Moreover, the expressions of MMP-2, MMP-7, and MMP-9 were significantly decreased, and the expression of TIMP-2 was dramatically increased in HTC116 cells after transfection with a miR-495 mimic. In addition, one key molecular step in the process of distant metastasis is characterized by the EMT. The activation of the EMT is observed in many types of malignant tumors, including CRC $[25,26]$. EMT causes the cancer cells to obtain invasive properties and metastatic growth characteristics. Genes such as E-cadherin, $\mathrm{N}$-cadherin, $\beta$-catenin, fibronection and vimentin have been established as the markers of EMT [27]. We assessed the changes in the EMT markers in HCT116 cells transfected with a miR-495 mimic and inhibitor. Our data demonstrated that the up-regulation of miR-495 could dramatically increase the level of the epithelial marker E-cadherin and decrease the mesenchymal markers $\beta$-catenin, fibronection and vimentin, which suggested that miR-495 might reverse the EMT process to suppress cell invasion and metastasis. Moreover, many transcription factors, such as Snail, Slug, Twist, ZEB1 and ZEB2, are considered crucial inducers of the EMT [28-30]. Further study showed that the introduction of miR-495 led to the down-regulation of the expression of Snail, Slug and ZEB1 and ZEB2. Collectively, our results indicated that overexpressing miR-495 inhibited the EMT through the downregulation of transcription factors, thereby suppressing cell invasion and metastasis of CRC.

Annexin A3 is a member of the annexin family, and it is important for diagnosis and prognosis. Several studies have reported malignancies associated with a high expression of Annexin A3, including prostate cancer [31], pancreatic cancer [32], kidney cancer [33], liver cancer [34], lung adenocarcinoma [35], ovarian cancer [36] and CRC [37], indicating that Annexin A3 may be a critical oncogene contributing to the metastasis of cancers. In the present study, Annexin A3 was also found to be up-regulated in CRC cells and tissues. Next, our findings confirmed that Annexin A3 is a direct target of miR-495 in CRC cells. Furthermore, we found that the knockdown of Annexin A3 also significantly inhibited the invasion and EMT of CRC cells, mimicking the effects of miR-495 overexpression. Additionally, the restoration of Annexin A3 partially blocked the inhibitory effects of the miR-495 mimic on the invasion and EMT of CRC cells, suggesting that Annexin A3 might play an important role in the metastasis of CRC. In addition, many studies have reported that down-stream of p53 is the EMT [38]. For example, the activation of p53 resulted in the increased repression of Snail, an EMT-inducing transcription factor [39]. G. Chiappetta et al. showed that the ectopic expression of PATZ1 in thyroid cancer cells activates the p53-dependent pathways opposing EMT and cell migration to prevent invasiveness [40]. Yan et al. reported that the overexpression of Annexin A3 inhibited the level of p53, thus enhancing the platinum-drug resistance in ovarian cancer cells [36]. Based on these results, we propose the hypothesis that Annexin A3 could modulate the EMT through the regulation of p53. After silencing p53, the effects of Annexin A3 knockdown on invasion and EMT were partially blocked. 


\section{Cellular Physiology Cell Physiol Biochem 2017;44:1882-1895 \begin{tabular}{l|l|l} 
and Biochemistry Published online: December 08, 2017 & $\begin{array}{l}\text { (c) } 2017 \text { The Author(s). Published by S. Karger AG, Basel } \\
\text { www.karger.com/cpb }\end{array}$
\end{tabular}}

Bai et al.: The Roles of MiR-495 in Colorectal Cancer

\section{Conclusion}

We demonstrated that the relative miR-495 expression was dramatically reduced in CRC tissues and cells. The up-regulation of miR-495 inhibited the invasion and EMT of CRC cells by the down-regulation of Annexin A3. This novel miR-495/Annexin A3/p53 axis might provide new insights into the molecular mechanisms underlying invasion and metastasis of CRC, and the overexpression of miR-495 might be a possible therapeutic strategy for the therapy of CRC in the future.

\section{Acknowledgements}

This work was supported by the Beijing Municipal Administration of Hospitals Clinical Medicine Development of Special Funding (ZYLX201504), National Key Technologies R\&D Program (No.2015BAI13B09), and Support Project of High-level Teachers in Beijing Municipal Universities in the Period of 13th Five-year Plan (IDHT20170516).

\section{Disclosure Statement}

None.

\section{References}

$>1$ Siegel RL, Miller KD, Jemal A: Cancer statistics, 2015. CA 2015;65, 5-29.

2 Siegel R, Naishadham D, Jemal A: Cancer statistics, 2012. CA 2012;62:10-29.

-3 Li Q Liang X, Wang Y, Meng X, Xu Y, Cai S, Wang Z, Liu J, Cai G: miR-139-5p Inhibits the EpithelialMesenchymal Transition and Enhances the Chemotherapeutic Sensitivity of Colorectal Cancer Cells by Downregulating BCL2. Sci Rep 2016;6:27157.

4 Din FV, Theodoratou E, Farrington SM, Tenesa A, Barnetson RA, Cetnarskyj R, Stark L, Porteous ME, Campbell H, Dunlop MG: Effect of aspirin and NSAIDs on risk and survival from colorectal cancer. Gut 2010;59:1670-1679.

5 Gerke V. Moss SE: Annexins: from structure to function. Physiol Rev 2002;82:331-371.

6 Hayes MJ, Moss SE: Annexins and disease. Biochem Biophys Res Commun 2004;322:1166-1170.

7 Lim LH, Pervaiz S: Annexin 1: the new face of an old molecule. FASEB J. 2007;21:968-975.

8 Rand JH: The annexinopathies: a new category of diseases. Biochim Biophys Acta 2000;1498:169-173.

9 Mussunoor S, Murray GI: The role of annexins in tumour development and progression. J Pathol 2008;216:131-140.

10 Martello G, Rosato A, Ferrari F, Manfrin A, Cordenonsi M, Dupont S, Enzo E, Guzzardo V, Rondina M, Spruce T, Parenti AR, Daidone MG, Bicciato S, Piccolo S: A microRNA targeting diacer for metastasis control. Cell 2010;141:1195-1207.

-11 Uematsu K, He B, You L, Xu Z, McCormick F, Jablons DM: Activation of the Wnt pathway in non small cell lung cancer: evidence of disheveled overexpression. Oncogene 2003;22:7218-7221.

12 Zhao S, Sun H, Jiang W, Mi Y, Zhang D, Wen Y, Cheng D, Tang H, Wu S, Yu Y, Liu X, Cui W, Zhang M, Sun X, Zhou Z, Peng Z, Yan D: miR-4775 promotes colorectal cancer invasion and metastasis via the Smad7/TGF $\beta$ mediated epithelial to mesenchymal transition. Mol Cancer 2017;16:12.

-13 Wei W, Yang Y, Cai J, Cui K, Li RX, Wang H, Shang X, Wei D: MiR-30a-5p Suppresses Tumor Metastasis of Human Colorectal Cancer by Targeting ITGB3. Cell Physiol Biochem 2016;39:1165-1176.

14 Zhou L, Xu Z, Ren X, Chen K, Xin S: MicroRNA-124 (MiR-124) Inhibits Cell Proliferation, Metastasis and Invasion in Colorectal Cancer by Downregulating Rho-Associated Protein Kinase 1(ROCK1). Cell Physiol Biochem 2016;38:1785-1795.

15 Zhang B, Yuan F, Liu J, Li Y, Zhou F, Liu X, Hao Z, Li Q Zheng Y, Wang W: Hsa-miR-495 acts as a tumor suppressor gene in glioma via the negative regulation of MYB. Mol Med Rep 2016;14:977-982.

16 Li Z, Cao Y, Jie Z, Liu Y, Li Y, Li J, Zhu G, Liu Z, Tu Y, Peng G, Lee DW, Park SS: miR-495 and miR-551a inhibit the migration and invasion of human gastric cancer cells by directly interacting with PRL-3. Cancer Lett 2012;323:41-47.

17 Xu YY, Tian J, Hao Q, Yin LR: MicroRNA-495 downregulates FOXC1 expression to suppress cell growth and migration in endometrial cancer. Tumour Biol 2016;37:239-251. 


\section{Cellular Physiology Cell Physiol Biochem 2017;44:1882-1895 \begin{tabular}{l|l|l} 
and Biochemistry Published onlIne: December 08, 2017 & $\begin{array}{l}\text { (c) } 2017 \text { The Author(s). Published by S. Karger AG, Basel } \\
\text { www.karger.com/cpb }\end{array}$ \\
\hline
\end{tabular}}

Bai et al.: The Roles of MiR-495 in Colorectal Cancer

18 Cao M, Nie W, Li J, Zhang Y, Yan X, Guan X, Chen X, Zen K, Zhang CY, Jiang X, Hou D: MicroRNA-495 induces breast cancer cell migration by targeting JAM-A. Protein Cell 2014;5:862-872.

19 Tan M, Mu X, Liu Z, Tao L, Wang J, Ge J, Qiu J: microRNA-495 promotes bladder cancer cell growth and invasion by targeting phosphatase and tensin homolog. Biochem Biophys Res Commun 2017;483:867-873.

-20 Jung EJ, Moon HG, Park ST, Cho BI, Lee SM, Jeong CY, Ju YT, Jeong SH, Lee YJ, Choi SK, Ha WS, Lee JS, Kang KR, Hong SC: Decreased annexin A3 expression correlates with tumor progression in papillary thyroid cancer. Proteomics Clin Appl 2010;4:528-437.

21 Welch JP, Donaldson G: The clinical correlation of an autopsy study of recurrent colorectal cancer. Ann Surg 1979;189:496-502.

22 Park JE, Lee DH, Lee JA, Park SG, Kim NS, Park BC, Cho S. Annexin A3 is a potential angiogenic mediator. Biochem Biophys Res Commun 2005;337:1283-1287.

23 Simpson-Haidaris PJ, Rybarczyk B: The role of fibrinogen as an extracellular matrix protein. Ann N Y Acad Sci 2001;936:406-425.

24 Li BH, Zhao P, Liu SZ, Yu YM, Han M, Wen JK: Matrix metalloproteinase-2 and tissue inhibitor of metalloproteinase-2 in colorectal carcinoma invasion and metastasis.World J Gastroenterol 2005;11:3046-3050.

25 Guarino M: Epithelial-mesenchymal transition and tumour invasion. Int J Biochem Cell Biol 2007;39:21532160.

26 Thiery JP, Acloque H, Huang RY, Nieto MA: Epithelial-mesenchymal transition in development and disease. Cell 2009;139:871-890.

27 Kalluri R, Weinberg RA: The basics of epithelial-mesenchymal transition. J Clin Invest 2009;119:1420-1428.

28 Khan MA, Chen HC, Zhang D, Fu J: Twist: a molecular target in cancer therapeutics. Tumor Biol 2013;34:2497-2506.

29 Peinado H, Olmeda D, Cano A: Snail, Zeb and bHLH factors in tumour progression: an alliance against the epithelial phenotype? Nat Rev Cancer 2007;7:415-428.

-30 De Craene B, Berx G: Regulatory networks defining EMT during cancer initiation and progression. Nat Rev Cancer 2013;13:97-110.

-31 Köllermann J, Schlomm T, Bang H, Schwall GP, von Eichel-Streiber C, Simon R, Schostak M, Huland H, Berg W, Sauter G, Klocker H, Schrattenholz A: Expression and prognostic relevance of annexin A3 in prostate cancer. Eur Urol 2008;54:1314-1323.

32 Baine MJ, Chakraborty S, Smith LM, Mallya K, Sasson AR, Brand RE, Batra SK: Transcriptional profiling of peripheral blood mononuclear cells in pancreatic cancer patients identifies novel genes with potential diagnostic utility. PLoS ONE 2011;6:e17014.

33 Bianchi C, Bombelli S, Raimondo F, Torsello B, Angeloni V, Ferrero S, Di Stefano V, Chinello C, Cifola I, Invernizzi L, Brambilla P, Magni F, Pitto M, Zanetti G, Mocarelli P, Perego RA: Primary cell cultures from human renal cortex and renal cell carcinoma evidence a differential expression of two spliced isoforms of annexin A3. Am J Pathol 2010;176:1660-1670

34 Liang RC, Neo JC, Lo SL, Tan GS, Seow TK, Chung MC: Proteome database of hepatocellular carcinoma. J Chromatogr B 2002;771:303-328

-35 Liu YF, Xiao ZQ, Li MX, Li MY, Zhang PF, Li C, Li F, Chen YH, Yi H, Yao HX, Chen ZC: Quantitative proteome analysis reveals annexin A3 as a novel biomarker in lung adenocarcinoma. J Pathol 2009;217:54-64

-36 Yan X, Yin J, Yao H, Mao N, Yang Y, Pan L: Increased expression of annexin A3 is a mechanism of platinum resistance in ovarian cancer. Cancer research 2010;70:1616-1624.

-37 Yip KT, Das PK, Suria D, Lim CR, Ng GH, Liew CC: A case-controlled validation study of a blood based seven gene biomarker panel for colorectal cancer in Malaysia. J Exp Clin Cancer Res 2010;29:128.

38 Wu CW, Peng ML, Yeh KT, Tsai YY, Chiang CC, Cheng YW: Inactivation of p53 in pterygium influence miR200a expression resulting in ZEB1/ZEB2 up-regulation and EMT processing. Exp Eye Res 2016;146:206211.

-39 Hünten S, Hermeking H: p53 directly activates cystatin D/CST5 to mediate mesenchymal-epithelial transition: a possible link to tumor suppression by vitamin D3. Oncotarget 2015; 6:15842-15856.

40 Chiappetta G, Valentino T, Vitiello M, Pasquinelli R, Monaco M, Palma G, Sepe R, Luciano A, Pallante P, Palmieri D, Aiello C, Rea D, Losito SN, Arra C, Fusco A, Fedele M: PATZ1 acts as a tumor suppressor in thyroid cancer via targeting p53-dependent genes involved in EMT and cell migration.Oncotarget 2015;6:5310-5323. 